Conference Proceedings Paper - 1st International Electronic Conference on Molecular Science:

Cell Signaling, Survival and Growth

\title{
Anti-Inflammatory Effect of Quercetin on RAW 264.7 Mouse Macrophages Induced with Polyinosinic-Polycytidylic Acid
}

\section{Young-Jin Kim and Wansu Park *}

Department of Pathology, College of Korean Medicine, Gachon University, Seong-nam, 13120, Republic of Korea; E-Mails: godsentry@naver.com

* Author to whom correspondence should be addressed; E-Mail: pws98@gachon.ac.kr; Tel.: +82-31-750-8821; Fax: +82-303-3447-2836.

Received: 19 September 2015 / Published: 13 October 2015

\begin{abstract}
Quercetin (3,3',4',5,6-Pentahydroxyflavone) is one of well-known antioxidants and a flavonol found in many fruits, leaves, and vegetables. Quercetin is also well-known to have anti-inflammatory effects on lipopolysaccharide-induced macrophages. However, the effects of quercetin on virus-induced macrophages are not fully reported. In this study, the anti-inflammatory effect of quercetin on double-stranded RNA (dsRNA)-induced macrophages was examined. Quercetin at concentrations of up to $50 \mu \mathrm{M}$ significantly inhibited the production of nitric oxide (NO), IL-6, MCP-1, IP-10, RANTES, GM-CSF, GCSF, TNF- $\alpha$, LIF, LIX, and VEGF as well as calcium release in dsRNA [50 $\mu \mathrm{g} / \mathrm{mL}$ of polyinosinic-polycytidylic acid]-induced RAW 264.7 mouse macrophages $(P<0.05)$. Quercetin at concentrations of up to $50 \mu \mathrm{M}$ also significantly inhibited mRNA expression of signal transducer and activated transcription 1 (STAT1) and STAT3 in dsRNA-induced RAW $264.7(P<0.05)$. In conclusion, quercetin has alleviating effects on viral inflammation concerned with its inhibition of NO, cytokines, chemokines, and growth factors in dsRNA-induced macrophages via the calcium-STAT pathway.
\end{abstract}

Keywords: quercetin; dsRNA; inflammation; macrophages; nitric oxide; cytokine; calcium; STAT 


\section{Introduction}

Immunity is essential for life. Bartemes et al. have reported that innate immunity provides the first line of response to invading pathogens and a variety of environmental insults [1]. Gilroy has reported that inflammation is a primordial response that protects against infection, cancer and tissue injury and restores damaged tissue to its normal physiological functioning; in fact, our wellbeing and survival depends upon inflammation's efficiency and carefully balanced control [2]. In recent study, Legatzki et al. have meaningfully reported that it is becoming more important to maintain inflammatory homeostasis and to induce immune regulation [3].

Macrophages are important in immunity. Eo et al. have reported that inflammation is an innate immune response by various immune cells including macrophages for the protection against the harmful stimuli such as virus and bacteria [4]. During inflammatory reaction, macrophages and monocytes produce inflammatory mediators such as nitric oxide (NO), cytokines, chemokines (chemotactic cytokines), and growth factors. And calcium release in macrophages levels up with inflammatory processes. Therefore, O'Shea et al. have reported that modulation of macrophagemediated inflammatory responses is important for creating a new therapeutic approach against inflammatory diseases [5].

Cho et al. have reported that virus can induce tissue necrosis (programmed) and inflammation; viral infections can activate immune responses, trigger inflammatory diseases, and promote cancer growth [6]. Lee and Park have reported that the double-stranded RNA (dsRNA), which accumulates at various stages of viral replication, stimulates macrophages; like other pathogenic endotoxin, dsRNA-activated macrophages provokingly produce many kinds of inflammatory mediators, including NO, cytokines, chemokines, and growth factors, resulting in acute or chronic inflammation; polyinosinic-polycytidylic acid (PIC) is considered as the synthetic analog of dsRNA [7].

Quercetin (Figure 1) a flavonol found in many fruits, vegetables, and leaves. Quercetin is known to be anti-oxidative [8]. Macrophages have been used to evaluate inflammatory activity of various materials [9-12]. However, the effects of quercetin on PIC-induced macrophages are not fully reported.<smiles>O=c1c(O)c(-c2ccc(O)c(O)c2)oc2cc(O)cc(O)c12</smiles>

Figure 1. Chemical structure of quercetin.

In the present study, we investigated the inhibitory effect of quercetin on PIC-induced inflammation using RAW 264.7 mouse macrophages. Finally, it was demonstrated that quercetin at concentrations of up to $50 \mu \mathrm{M}$ significantly inhibited the production of nitric oxide (NO), interleukin (IL)-6, tumor necrosis factor (TNF)- $\alpha$, monocyte chemotactic protein (MCP)-1, interferon inducible protein-10 (IP-10), RANTES (CCL5), lipopolysaccharide-induced CXC chemokine (LIX; CXCL5), leukemia inhibitory factor (LIF; IL-6 class cytokine), granulocyte colony-stimulating factor (G-CSF), granulocyte macrophage colony-stimulating factor (GM-CSF), and vascular endothelial growth factor (VEGF) as 
well as calcium release and mRNA expression of Signal Transducers and Activators of Transcription 1 (STAT1) and STAT3 in PIC-induced RAW 264.7 mouse macrophages $(P<0.05)$.

\section{Results}

\subsection{Effect of quercetin on NO production}

$\mathrm{NO}$ is one of major inflammatory mediators in immuno-inflammatory reaction. NO concentration in culture medium could be determined by the Griess reaction. In this study, after incubation of cells with materials for $24 \mathrm{~h}, 100 \mu \mathrm{L}$ of supernatant from each well was mixed with $100 \mu \mathrm{L}$ of Griess reagent in wells of a 96-well plate for determining NO concentration. Data represented that quercetin significantly inhibits excessive production of NO in PIC-induced RAW 264.7 mouse macrophages (Figure 2).

\subsection{Effect of quercetin on intracellular calcium release}

Intracellular calcium release is related with many cellular processes including inflammatory reaction. Calcium release in RAW 264.7 mouse macrophages could be determined by Fluo-4 assay. In this study, after incubation of cells with materials for $18 \mathrm{~h}$, the medium was removed and cells were incubated with the Fluo-4 dye loading solution. After incubation, the fluorescence intensity of each well was determined spectrofluorometrically with excitation and emission filters of $485 \mathrm{~nm}$ and 535 $\mathrm{nm}$, respectively. Data showed that quercetin significantly inhibits the calcium release in PIC-induced RAW 264.7 mouse macrophages (Figure 2).
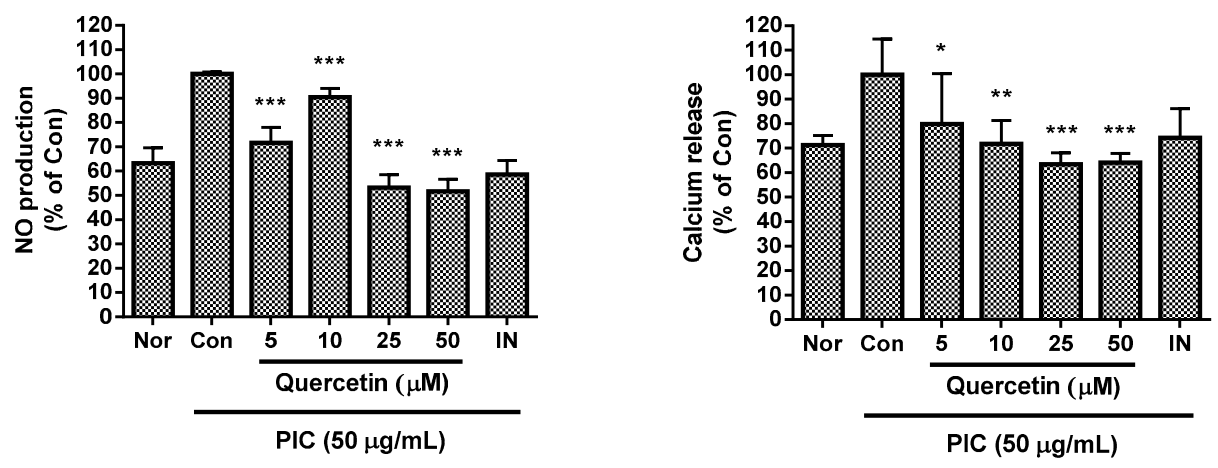

Figure 2. Effect of quercetin on NO production and calcium release in dsRNA-induced RAW 264.7 mouse macrophages. After $24 \mathrm{~h}$ treatment, NO production was measured by the Griess reaction assay. Calcium release was measured with Fluo-4 calcium assay after $18 \mathrm{~h}$ treatment. Normal group (Nor) was treated with media only. Control group (Con) was treated with the synthetic analog of dsRNA (PIC, $50 \mu \mathrm{g} / \mathrm{mL}$ of polyinosinic-polycytidylic acid) alone. IN denotes indomethacin $(0.5 \mu \mathrm{M})$. Values are the mean \pm SD of three independent experiments. $* P<0.05$ vs. Con; ** $P<0.01$; *** $P<0.001$. 

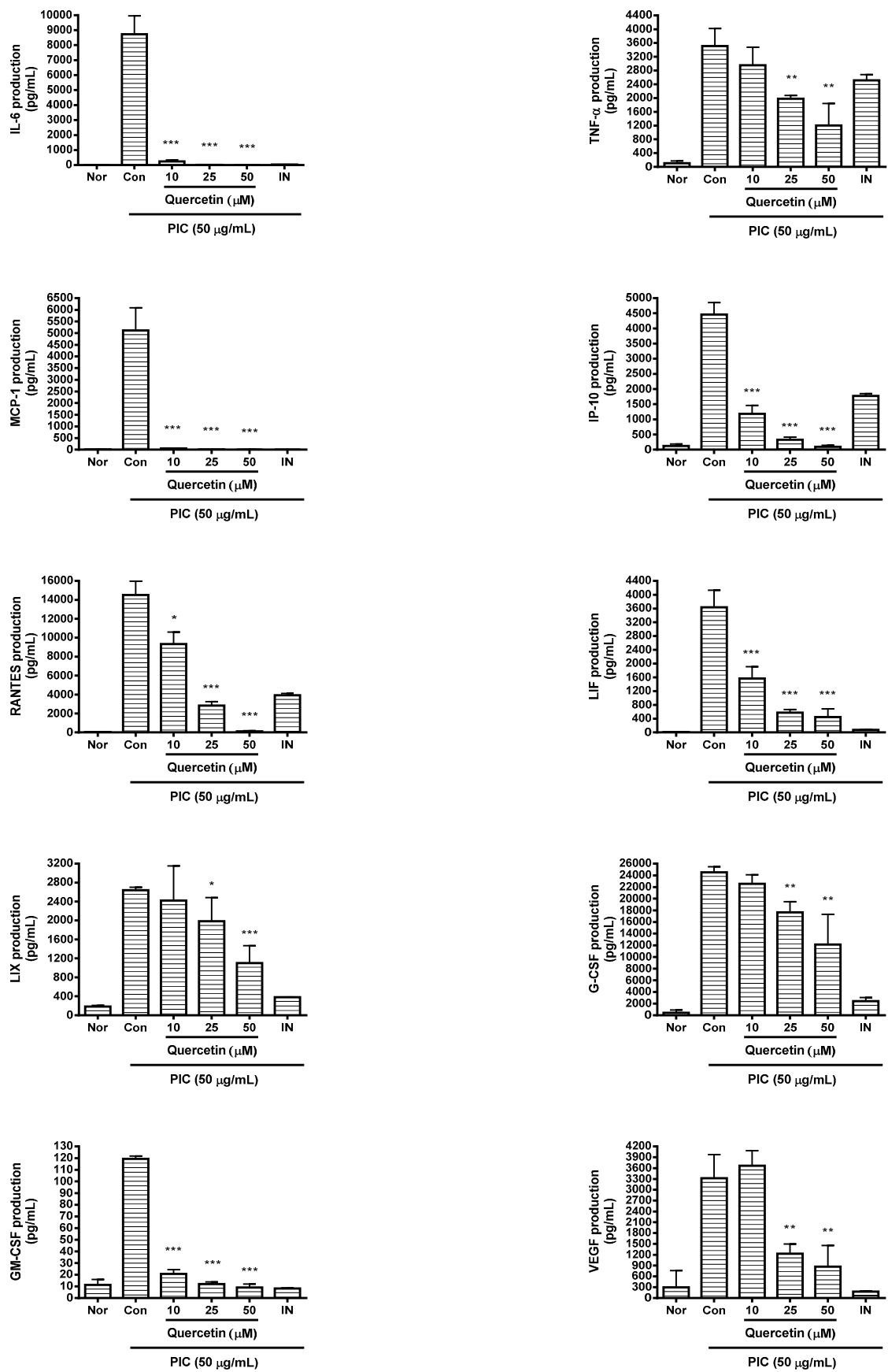

Figure 3. Effect of quercetin on production of cytokines such as IL-6, TNF- $\alpha$, MCP-1, IP10, RANTES, LIF, LIX, G-CSF, GM-CSF, and VEGF in dsRNA-induced RAW 264.7 mouse macrophages. Flourescene intensity of each cytokine in the culture medium was measured by a Multiplex bead-based cytokine assay after $24 \mathrm{~h}$ incubation. Normal group (Nor) was treated with media only. Control group (Con) was treated with the synthetic analog of dsRNA (PIC, $50 \mu \mathrm{g} / \mathrm{mL}$ of polyinosinic-polycytidylic acid) alone. IN denotes indomethacin $(0.5 \mu \mathrm{M})$. Values are the mean \pm SD of three independent experiments. ${ }^{*} P<$ 0.05 vs. Con; ** $P<0.01 ; * * * P<0.001$. 
Cytokines are a distinctive category of small protein $(5 \sim 20 \mathrm{kDa})$ that are important in inflammation and cell signaling. Cytokine production in culture medium could be determined by multiplex cytokine assay. In this study, after incubation of cells with materials for $24 \mathrm{~h}$, cytokines released from incubated cells were measured in cell culture supernatants using a Luminex assay based on xMAP technology. Data represented that quercetin significantly reduces excessive productions of IL-6, TNF- $\alpha$, MCP-1, IP-10, RANTES, LIF, LIX, G-CSF, GM-CSF, and VEGF in PIC-induced RAW 264.7 mouse macrophages (Figure 3).

\subsection{Effect of quercetin on mRNA expression of STAT1 and STAT3}

STAT proteins are regarded to play an important role in inflammatory reaction. In this study, real time RT-PCR was performed to determine mRNA expression of STAT1 (GenBank: NM_009283) and STAT3 (GenBank: NM_213659) after incubation of cells with materials for $18 \mathrm{~h}$. The GAPDH gene (GenBank: NM_001001303) was used for RNA normalization. Data represented that quercetin significantly inhibited mRNA expression of STAT1 and STAT3 in PIC-induced RAW 264.7 mouse macrophages (Figure 4). This means that quercetin modulates inflammatory reactions in PIC-induced macrophages via the calcium-STAT pathway.
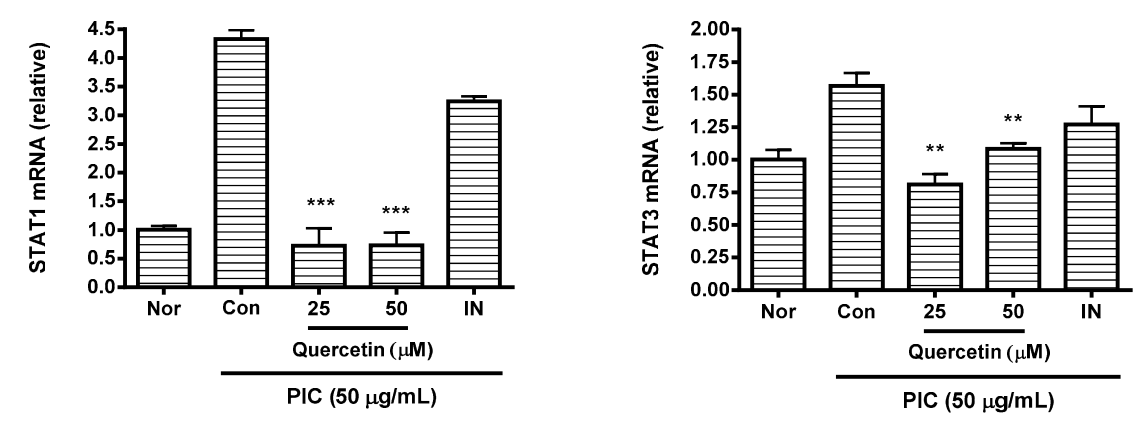

Figure 4. Effect of quercetin on mRNA expression of STAT1 and STAT3 in dsRNAinduced RAW 264.7 mouse macrophages. After $18 \mathrm{~h}$ treatment, the mRNA expression of STAT1 and STAT3 was measured by real time RT-PCR assay. STAT1 and STAT3 mRNA were normalized to the housekeeping gene GAPDH mRNA. Normal group (Nor) was treated with media only. Control group (Con) was treated with the synthetic analog of dsRNA (PIC, $50 \mu \mathrm{g} / \mathrm{mL}$ of polyinosinic-polycytidylic acid) alone. IN denotes indomethacin $(0.5 \mu \mathrm{M})$. Values are the mean \pm SD of three independent experiments. $* * P$ $<0.01$ vs. Con; *** $P<0.001$.

\section{Discussion}

Quercetin is known to be anti-oxidative [8]. Hollman et al. have reported that quercetin, a dietary antioxidant flavonoid, has anticarcinogenic properties [13]. Chen and Pace-Asciak have reported that quercetin dose-dependently inhibits the contractile response tonoradrenaline in isolated endothelium-intact rat aorta [14]. However, the effects of quercetin on PIC-induced macrophages are not fully reported. 
Gadient and Patterson have reported that inflammation is a general term used to describe the many diverse processes that tissues employ in response to infections by pathogens (such as bacteria, virus, parasites, and fungi) and injuries caused by toxic molecules or physical damage such as burns or cuts [15]. Lee et al. have reported that inflammation plays a critical defensive role in the human body; however, uncontrolled or aberrant inflammatory responses contribute to various acute and chronic inflammatory diseases [16].

Smith has reported that viral infections trigger anti-viral type I interferon responses by stimulating endosomal and cytosolic pattern recognition receptors including Toll-like receptors, key initiators of inflammation [17]. Mahima et al. have reported that there are ongoing trends of immunomodulation to combat a vast range of human and animal diseases including the incurable diseases like viral diseases, cancers, autoimmune diseases and inflammatory conditions [18]. Cooper et al. have reported that viral or bacterial infections induce exacerbations and increase the morbidity of patients with chronic airways diseases; a viral infection triggers an innate immune response through host detection of viral dsRNA via several different mechanisms, including activation of a member of the class of pattern recognition receptors, Toll-like receptor 3; lung inflammation associated with viral infections includes the robust increase in cytokines such as IL-1 $\beta$, IL-6, IL-8, TNF- $\alpha$, GM-CSF, IP-10, RANTES, and MIP-1 $\alpha$ [19].

Kang et al. have reported that, among the many immuno-inflammatory leukocytes, macrophages and monocytes are of great importance [20]. Cammer and Cox have reported that macrophages, which are organized throughout every tissue, represent a key component of the immune system and the recruitment of macrophages to specific sites is important in normal host defense [21]. Medina et al. have reported that macrophages play an important role in inflammatory disease through the release of factors such as NO, reactive oxygen species, inflammatory cytokines, chemokines, growth factors, and prostaglandin mediators involved in the immune response [22].

Oates has reported that NO is a membrane-permeable signaling molecule involved in a broad array of biologic processes through its ability to modify proteins, lipids, and DNA and alter their function and immunogenicity [23]. Karpuzoglu and Ahmed have reported that appropriate levels of NO assist in mounting an effective defense against invading microbes; conversely, inability to generate NO results in serious, even fatal, susceptibility to infections; further, dysregulation or overproduction of NO has been implicated in the pathogenesis of many disorders, including atherosclerosis, neurodegenerative diseases, inflammatory autoimmune diseases, and cancer; therefore, depending upon the levels of NO generated, the potential exists for NO to behave like a "double-edged" biological sword; taking these issues into consideration, it is thus pivotal to understand the regulation of NO [24].

Inflammasomes is inflammatory multiprotein complexes. Hagar and Miao have reported that inflammasomes detect signatures of microbial infection and trigger caspase-1 or caspase-11 activation, culminating in cytokine secretion and obliteration of the replicative niche via pyroptosis [25]. Nazemian et al. have reported that overproduction of inflammatory cytokines can induce complications such as atherosclerosis, malnutrition and anaemia, which are mostly resistant to erythropoietin treatment [26]. Sipos et al. have reported that if infectious pathogens cannot be eliminated, they may elicit chronic inflammation, which has long been suggested to provoke genetic mutations and epigenetic mechanisms that promote malignant cell transformation [27]. Allavena et al. have reported that inflammatory conditions affecting organs such as liver, pancreas, stomach, colon, and prostate are associated with increased risk of cancer; cancer-associated inflammation includes 
leukocyte infiltration, prominently tumor-associated macrophages; expression of cytokines such as TNF or IL-1, chemokines such as MCP-1 (CCL2), tissue remodelling and angiogenesis [28]. Gorbachev and Fairchild have reported that chemokines are chemotactic cytokines critical for homeostatic and inflammation-induced trafficking of leukocytes during immune responses, hematopoesis, wound healing, and tumorigenesis [29]. Recently, Choi et al. have reported that VEGF is supposed to contribute to the pathogenesis of allergic airway disease [30]. McKinley et al. have reported that level of G-CSF increases in allergic airway inflammation [31]. Waring et al. have reported that LIF is implicated as a potential mediator of the local or systemic inflammatory response or the joint destruction seen in inflammatory arthritis [32]. Ruddy et al. have reported that LIX expression increases in inflammatory bone diseases such as arthritis and periodontal disease [33].

The present study shows that quercetin might alleviate PIC-induced inflammation by decreasing levels of inflammatory mediators such as NO, cytokines, chemokines, and growth factors in RAW 264.7 mouse macrophages; quercetin can be a candidate material for treating virus-concerned inflammatory diseases.

Timmins et al. have reported that in oxidative stress, the endoplasmic reticulum (ER) calcium stores are reduced and intracellular calcium concentration is increased, resulting in ER stress-mediated STAT1 activation; preapoptotic ER stress itself has proinflammatory effects in macrophages [34]. In the present study, quercetin significantly inhibits calcium release and mRNA expression of STAT1 and STAT3 in PIC-induced RAW 264.7 mouse macrophages. Thus, quercetin might modulate PICinduced macrophage activation via calcium-STAT pathway.

Finally, the present study demonstrates that quercetin has anti-inflammatory effect related with its inhibition of NO, IL-6, TNF- $\alpha$, MCP-1, IP-10, RANTES, LIF, LIX, G-CSF, GM-CSF, and VEGF production in PIC-induced macrophages via the calcium-STAT pathway. Further study is needed to evaluate medicinal usefulness of quercetin on viral inflammation.

\section{Experimental Section}

\subsection{Materials}

DMEM, FBS, penicillin, streptomycin, PBS, and other tissue culture reagents were purchased from Gibco BRL (Grand Island, NY, USA). Quercetin, indomethacin, griess reagent, and all other chemicals were purchased from Sigma-Aldrich (St. Louis, MO, USA).

\subsection{Quantification of NO production}

NO concentration in culture medium was determined by the Griess reaction. Specifically, after incubation of cells with materials for $24 \mathrm{~h}, 100 \mu \mathrm{L}$ of supernatant from each well was mixed with 100 $\mu \mathrm{L}$ of Griess reagent in wells of a 96-well plate. After an incubation of $15 \mathrm{~min}$ at room temperature, OD was determined at $540 \mathrm{~nm}$ with a microplate reader (Bio-Rad, Hercules, CA, USA) [7].

\subsection{Intracellular calcium assay}

After RAW 264.7 mouse macrophages were seeded in wells of 96-well plates, materials were added to the culture medium and incubation was carried out for $18 \mathrm{~h}$ at $37^{\circ} \mathrm{C}$. Thereafter, the medium was 
removed and cells were incubated with $100 \mu \mathrm{L}$ of the Fluo-4 dye loading solution (Molecular Probes, Eugene, OR, USA) for $30 \mathrm{~min}$ at $37^{\circ} \mathrm{C}$. After incubation, the fluorescence intensity of each well was determined spectrofluorometrically (Dynex, West Sussex, UK) with excitation and emission filters of $485 \mathrm{~nm}$ and $535 \mathrm{~nm}$, respectively [9].

\subsection{Multiplex bead-based cytokine assay}

After $24 \mathrm{~h}$ treatment with materials, cytokines released from treated cells were measured in cell culture supernatants using a Luminex assay based on xMAP technology. This assay was performed with Milliplex kits (Millipore, Billerica, MA, USA) and Bio-Plex 200 suspension array system (BioRad) as described previously [10-12]. Standard curves for each cytokine were generated using the kitsupplied reference cytokine samples.

\subsection{RNA isolation and real time RT-PCR analysis}

At the end of $18 \mathrm{~h}$ incubation with materials, RAW 264.7 mouse macrophages were lysed and Total RNA was isolated using the NucleoSpin RNA kit (Macherey-Nagel, Duren, Germany) according to the manufacturer's instructions. RNA quantity and quality were confirmed using Experion RNA StdSens Analysis kit (Bio-Rad) and Experion Automatic Electrophoresis System (Bio-Rad). cDNA was synthesized from $1 \mu \mathrm{g}$ total RNA using iScript cDNA Synthesis kit (Bio-Rad). Real time RT-PCR was performed using the iQ SYBR Green Supermix (Bio-Rad). The GAPDH gene was used for RNA normalization. Analysis was performed on a Bio-Rad CFX 96 Real-Time PCR Detection System (BioRad). Relative changes in the gene expression were calculated using the comparative threshold cycle (Ct) method (Bio-Rad) [12]. The following primers were used: STAT1 (GenBank: NM_009283), sense: 5'-TGAGATGTCCCGGATAGTGG-3'， antisense: 5'-CGCCAGAGAGAAATTCGTGT-3'; STAT3 (GenBank: NM_213659), sense: 5'-GTCTGCAGAGTTCAAGCACCT-3', antisense: 5'TCCTCAGTCACGATCAAGGAG-3'; GAPDH (GenBank: NM_001001303), sense: 5'AACCTGCCAAGTATGATGAC-3', antisense: 5'-GGGAGTTGCTGTTGAAGT-3'.

\subsection{Statistical analysis}

The results shown are summarized from three independent experiments and represent the mean \pm SD. Significant differences were examined using a Student's $t$-test with SPSS 11.0 software (SPSS, Chicago, IL, USA). In all cases, a $P$ value $<0.05$ was considered significant.

\section{Acknowledgments}

The author would like to thank Ji Young Lee and Hyun Joo Kim (College of Korean Medicine, Gachon University) for excellent technical assistance.

\section{Author Contributions}

Young-Jin Kim did experiments. Wansu Park analyzed data and prepared the manuscript.

\section{Conflicts of Interest}


The authors declare no conflict of interest.

\section{References and Notes}

1. Bartemes, K.R.; Iijima, K.; Kobayashi, T.; Kephart, G.M.; McKenzie, A.N.; Kita, H. IL-33Responsive Lineage- CD25+ CD44(Hi) Lymphoid Cells Mediate Innate Type 2 Immunity and Allergic Inflammation in the Lungs. J. Immunol. 2012, 188, 1503-1513.

2. Gilroy, D.W. Eicosanoids and the Endogenous Control of Acute Inflammatory Resolution. Int. J. Biochem. Cell Biol. 2010, 42, 524-528.

3. Legatzki, A.; Rösler, B.; von Mutius, E. Microbiome Diversity and Asthma and Allergy Risk. Current Allergy and Asthma Reports 2014, 14, 1-9.

4. Eo, H.J.; Park, J.H.; Park, G.H.; Lee, M.H.; Lee, J.R.; Koo, J.S.; Jeong, J.B. Anti-Inflammatory and Anti-Cancer Activity of Mulberry (Morus Alba L.) Root Bark. BMC complementary and alternative medicine 2014, 14, 200.

5. O'Shea, J.J.; Ma, A.; Lipsky, P. Cytokines and Autoimmunity. Nature Reviews Immunology 2002, 2, 37-45.

6. Cho, Y.; Challa, S.; Moquin, D.; Genga, R.; Ray, T.D.; Guildford, M.; Chan, F.K. Phosphorylation-Driven Assembly of the RIP1-RIP3 Complex Regulates Programmed Necrosis and Virus-Induced Inflammation. Cell 2009, 137, 1112-1123.

7. Lee, J.Y.; Park, W. Anti-Inflammatory Effect of Myristicin on RAW 264.7 Macrophages Stimulated with Polyinosinic-Polycytidylic Acid. Molecules 2011, 16, 7132-7142.

8. Anjaneyulu, M.; Chopra, K. Quercetin, an anti-oxidant Bioflavonoid, Attenuates Diabetic Nephropathy in Rats. Clinical and Experimental pharmacology and physiology 2004, 31, 244-248.

9. Lee, J.Y.; Park, W.; Yi, D.K. Immunostimulatory Effects of Gold Nanorod and Silica-Coated Gold Nanorod on RAW 264.7 Mouse Macrophages. Toxicol. Lett. 2012, 209, 51-57.

10. Yoon, S.; Lee, Y.; Park, S.K.; Kim, H.; Bae, H.; Kim, H.M.; Ko, S.; Choi, H.Y.; Oh, M.S.; Park, W. Anti-Inflammatory Effects of Scutellaria Baicalensis Water Extract on LPS-Activated RAW 264.7 Macrophages. J. Ethnopharmacol. 2009, 125, 286-290.

11. Yuk, S.S.; Lim, E.M.; Lee, J.Y.; Lee, Y.J.; Kim, Y.S.; Lee, T.H.; Park, S.K.; Bae, H.; Kim, H.M.; Ko, S.G. et al. Antiinflammatory Effects of Epimedium Brevicornum Water Extract on Lipopolysaccharide-Activated RAW264.7 Macrophages. Phytother. Res. 2010, 24, 1781-1787.

12. Lee, J.Y.; Park, W. Anti-Inflammatory Effect of Wogonin on RAW 264.7 Mouse Macrophages Induced with Polyinosinic-Polycytidylic Acid. Molecules 2015, 20, 6888-6900.

13. Hollman, P.C.; van Trijp, J.M.; Mengelers, M.J.; de Vries, J.H.; Katan, M.B. Bioavailability of the Dietary Antioxidant Flavonol Quercetin in Man. Cancer Lett. 1997, 114, 139-140.

14. Chen, C.K.; Pace-Asciak, C.R. Vasorelaxing Activity of Resveratrol and Quercetin in Isolated Rat Aorta. General Pharmacology: The Vascular System 1996, 27, 363-366.

15. Gadient, R.A.; Patterson, P.H. Leukemia Inhibitory Factor, Interleukin 6, and Other Cytokines using the GP130 Transducing Receptor: Roles in Inflammation and Injury. Stem Cells 1999, 17, $127-137$. 
16. Lee, J.H.; Lee, K.; Su, Z.; Boyanapalli, S.S.; Barman, D.N.; Huang, M.; Chen, L.; Magesh, S.; $\mathrm{Hu}, \mathrm{L} . ;$ Kong, A.T. In Vitro and in Vivo Anti-Inflammatory Effects of a Novel 4, 6-Bis ((E)-4Hydroxy-3-Methoxystyryl)-1-Phenethylpyrimidine-2 (1 H)-Thione. Chem. Res. Toxicol. 2013, 27, $34-41$.

17. Smith, J.A. A New Paradigm: Innate Immune Sensing of Viruses Via the Unfolded Protein Response. Frontiers in microbiology 2014, 5.

18. Mahima; Ingle, A.M.; Verma, A.K.; Tiwari, R.; Karthik, K.; Chakraborty, S.; Deb, R.; Rajagunalan, S.; Rathore, R.; Dhama, K. Immunomodulators in Day to Day Life: A Review. Pak. J. Biol. Sci. 2013, 16, 826-843.

19. Cooper, P.R.; Lamb, R.; Day, N.D.; Branigan, P.J.; Kajekar, R.; San Mateo, L.; Hornby, P.J.; Panettieri, R.A.,Jr. TLR3 Activation Stimulates Cytokine Secretion without Altering Agonist-Induced Human Small Airway Contraction Or Relaxation. Am. J. Physiol. Lung Cell. Mol. Physiol. 2009, 297, L530-L537.

20. Kang, X.; Kim, H.J.; Ramirez, M.; Salameh, S.; Ma, X. The Septic Shock-Associated IL-10 -1082 A > G Polymorphism Mediates Allele-Specific Transcription Via Poly(ADP-Ribose) Polymerase 1 in Macrophages Engulfing Apoptotic Cells. J. Immunol. 2010, 184, 3718-3724.

21. Cammer, M.; Cox, D. Chemotactic Responses by Macrophages to a Directional Source of a Cytokine Delivered by a Micropipette. In Cytokine Bioassays; Springer: Berlin, Germany, 2014, pp. 125-135.

22. Medina, E.A.; Morris, I.R.; Berton, M.T. Phosphatidylinositol 3-Kinase Activation Attenuates the TLR2-Mediated Macrophage Proinflammatory Cytokine Response to Francisella Tularensis Live Vaccine Strain. J. Immunol. 2010, 185, 7562-7572.

23. Oates, J.C. The Biology of Reactive Intermediates in Systemic Lupus Erythematosus. Autoimmunity 2010, 43, 56-63.

24. Karpuzoglu, E.; Ahmed, S.A. Estrogen Regulation of Nitric Oxide and Inducible Nitric Oxide Synthase (iNOS) in Immune Cells: Implications for Immunity, Autoimmune Diseases, and Apoptosis. Nitric Oxide 2006, 15, 177-186.

25. Hagar, J.A.; Miao, E.A. Detection of Cytosolic Bacteria by Inflammatory Caspases. Curr. Opin. Microbiol. 2014, 17, 61-66.

26. Nazemian, F.; Karimi, G.; Moatamedi, M.; Charkazi, S.; Shamsara, J.; Mohammadpour, A.H. Effect of Silymarin Administration on TNF- $\alpha$ Serum Concentration in Peritoneal Dialysis Patients. Phytotherapy Research 2010, 24, 1654-1657.

27. Sipos, F.; Füri, I.; Constantinovits, M.; Tulassay, Z.; Müzes, G. Contribution of TLR Signaling to the Pathogenesis of Colitis-Associated Cancer in Inflammatory Bowel Disease. World journal of gastroenterology: WJG 2014, 20, 12713.

28. Allavena, P.; Garlanda, C.; Borrello, M.G.; Sica, A.; Mantovani, A. Pathways Connecting Inflammation and Cancer. Curr. Opin. Genet. Dev. 2008, 18, 3-10.

29. Gorbachev, A.V.; Fairchild, R.L. Regulation of Chemokine Expression in the Tumor Microenvironment. Critical Reviews ${ }^{\mathrm{TM}}$ in Immunology 2014, 34.

30. Choi, Y.H.; Jin, G.Y.; chang Li, L.; Yan, G.H. Inhibition of Protein Kinase C Delta Attenuates Allergic Airway Inflammation through Suppression of PI3K/Akt/mTOR/HIF-1 alpha/VEGF Pathway. 2013. 
31. McKinley, L.; Alcorn, J.F.; Peterson, A.; Dupont, R.B.; Kapadia, S.; Logar, A.; Henry, A.; Irvin, C.G.; Piganelli, J.D.; Ray, A. et al. TH17 Cells Mediate Steroid-Resistant Airway Inflammation and Airway Hyperresponsiveness in Mice. J. Immunol. 2008, 181, 4089-4097.

32. Waring, P.; Carroll, G.; Kandiah, D.; Buirski, G.; Metcalf, D. Increased Levels of Leukemia Inhibitory Factor in Synovial Fluid from Patients with Rheumatoid Arthritis and Other Inflammatory Arthritides. Arthritis \& Rheumatism 1993, 36, 911-915.

33. Ruddy, M.J.; Shen, F.; Smith, J.B.; Sharma, A.; Gaffen, S.L. Interleukin-17 Regulates Expression of the CXC Chemokine LIX/CXCL5 in Osteoblasts: Implications for Inflammation and Neutrophil Recruitment. J. Leukoc. Biol. 2004, 76, 135-144.

34. Timmins, J.M.; Ozcan, L.; Seimon, T.A.; Li, G.; Malagelada, C.; Backs, J.; Backs, T.; Bassel-Duby, R.; Olson, E.N.; Anderson, M.E. et al. Calcium/calmodulin-Dependent Protein Kinase II Links ER Stress with Fas and Mitochondrial Apoptosis Pathways. J. Clin. Invest. 2009, 119, 2925-2941.

(C) 2015 by the authors; licensee MDPI, Basel, Switzerland. This article is an open access article distributed under the terms and conditions of the Creative Commons Attribution license (http://creativecommons.org/licenses/by/4.0/). 